Meta

Journal des traducteurs

Translators' Journal

\title{
Les syntagmes nominaux complexes en anglais et en français : éléments de réflexion
}

\section{Marie-Josée Jastrab de Saint-Robert}

Volume 32, numéro 3, septembre 1987

La fertilisation terminologique dans les langues romanes

URI : https://id.erudit.org/iderudit/003261ar

DOI : https://doi.org/10.7202/003261ar

Aller au sommaire du numéro

Éditeur(s)

Les Presses de l'Université de Montréal

ISSN

0026-0452 (imprimé)

1492-1421 (numérique)

Découvrir la revue

Citer cet article

Jastrab de Saint-Robert, M.-J. (1987). Les syntagmes nominaux complexes en anglais et en français : éléments de réflexion. Meta, 32(3), 260-266.

https://doi.org/10.7202/003261ar d'utilisation que vous pouvez consulter en ligne. 


\title{
LES SYNTAGMES NOMINAUX COMPLEXES EN ANGLAIS ET EN FRANÇAIS : ÉLÉMENTS DE RÉFLEXION
}

\author{
MARIE-JoséE JASTRAB DE SAINT-ROBERT
}

New York University, USA

Cet article se propose d'aborder le problème de l'incidence de l'anglais ${ }^{1}$ sur le français par le biais de l'étude de la formation des syntagmes nominaux complexes dans ces deux langues. Sont considérés comme des syntagmes nominaux complexes les mots composés lexicalisés ou en voie de lexicalisation et les séquences nominales produites par les règles syntactiques ou sémantiques mais qui ne seraient pas présentes telles quelles dans le lexique. Parmi les mots composés nous retiendrons en particulier les mots composés complexes, tels que :

(1) a. input/output/device organe entrée/sortie

c. cash flow

b. split screen écran multifenêtre marge brute d'autofinancement

d. voice changer modulateur de voix

Les séquences nominales ordinaires qui nous intéresseront ici seront essentiellement des séquences composées de noms qualifiant d'autres noms :
(2) a. customer information
b. letter quality printer

\section{bureau}

\section{bureau d'information-client imprimante qualité courrier}

Du point de vue terminologique, ces deux types de syntagmes qui correspondent à des unités de syntaxe et de signification constituent des termes à part entière. Plusieurs questions seront au centre de notre réflexion : y-a-t-il influence de l'anglais sur le français dans le domaine de la formation de ces syntagmes ? N'y-a-t-il pas en français des règles susceptibles de produire de tels énoncés? Autrement dit, peut-on véritablement parler de fertilisation terminologique du français par l'anglais dans le domaine des constructions nominales?

Pour répondre à ces questions il nous faut tout d'abord définir ce que nous entendons par fertilisation terminologique. Cette notion peut en effet recouvrir différentes réalités. Ainsi, la fertilisation terminologique peut être considérée comme synonyme de fertilisation notionnelle. Comme la démarche terminologique a pour point de départ la notion et non le terme, il y a fertilisation terminologique dès lors qu'il y a importation d'une notion nouvelle exprimée d'abord en anglais puis reprise en français. Le syntagme research and development ( $R$ and $D$ ), d'abord apparu en anglais dans la terminologie militaire, de même que la notion qu'il exprime, a donné lieu à nombre d'équivalents français : élaboration et mise au point, étude et mise au point, recherche et mise au point avant de devenir recherche-développement (la R-D). Ce type de fertilisation terminologique est d'autant plus difficile à repérer que les spécialistes du domaine ne mentionnent pas toujours la source ou la langue source des notions qu'ils manipulent. Le terminologue doit donc consulter ou être lui-même un spécialiste du domaine pour pouvoir déter- 
miner avec précision s'il y a fertilisation notionnelle et donc terminologique en provenance de l'anglais.

Une seconde définition de la fertilisation terminologique peut être proposée pour mettre l'accent sur l'expansion du vocabulaire français sous l'influence du vocabulaire anglais en raison essentiellement de la reproduction en français de termes anglais ou d'inspiration anglaise. Les termes français ainsi créés seraient d'autant plus facilement repérables qu'ils entraîneraient une modification du français. Ainsi en est-il de l'expression armes conventionnelles qui reprend le terme anglais conventional armament tandis que tombe en désuétude son équivalent français armes classiques.

Une troisième approche de la fertilisation terminologique s'attache à relever non plus la multiplication de certains types de termes sous l'influence de l'anglais mais la multiplication de certains types de termes sous l'influence de l'anglais. Une telle démarche part des interrogations suivantes : certaines règles de formation de ces termes sontelles plus utilisées que d'autres? De nouvelles règles sont-elles appparues ? C'est essentiellement ces trois aspects du phénomène de la fertilisation terminologique que nous garderons à l'esprit en essayant de déterminer, d'une manière très fragmentaire certes, l'incidence de l'anglais sur la morphologie et la syntaxe du français. $\hat{A}$ cette fin, nous confronterons les arguments sur lesquels reposent deux hypothèses de travail : l'hypothèse de l'anglicisation du français et l'hypothèse de la ressemblance structurelle et sémantique de l'anglais et du français.

L'hypothèse de l'anglicisation du français se fonde tout d'abord sur la présence dans la langue française de syntagmes directement empruntés à l'anglais ou décalqués sur l'anglais.

En l'absence de données statistiques sur l'ampleur du phénomène de l'emprunt en français de syntagmes nominaux complexes anglais, et d'un accord sur une définition opérationnelle de l'emprunt, nous retiendrons ici deux exemples de fertilisation notionnelle ayant donné lieu à des emprunts directs en français :
(3) a. leasure wear
b. sportswear

qui sont entrés dans le vocabulaire français en raison des difficultés de traduction qu'ils posent en français. Wear dans ces deux cas ne correspond pas exactement au mot vêtement comme proposent de le traduire tous les dictionnaires bilingues. Parler de vêtements de loisir ou de vêtements de sport serait donc en partie inexact étant donné que les magasins de sportswear par exemple vendent également des chaussures de sport. L'emprunt direct à l'anglais s'explique ici par le fait que le terme français ne possède pas de terme générique alors que l'anglais dispose grâce à wear d'un hyperonyme qui entre dans la composition d'un certain nombre de locutions : men's wear, women's wear, evening wear, spring wear, country wear, foot wear, etc., rendues en français par différentes constructions syntaxiques. Il est à noter que la Commission générale de terminologie au sein du Commissariat général de la langue française qui recherche à l'heure actuelle un équivalent compréhensible en français ne se borne pas à traduire le terme anglais sportswear en s'inspirant des définitions qu'offrent les dictionnaires anglais et américains de ce terme (Webster propose une définition où il est expressément question de clothing, et donc de vêtement), mais tient à cerner aussi complètement que possible la notion telle qu'elle s'est implantée en milieu francophone.

Parfois un emprunt donne lieu à toute une série d'emprunts dérivés. Dans le vocabulaire boursier, par exemple nous trouvons :

(4) a. market making et market makers

b. pays prices makers et pays price takers

Un second argument avancé par l'hypothèse de l'anglicisation du français dans le domaine des syntagmes nominaux complexes se fonde sur le parallélisme de ces cons- 
tructions dans les deux langues. L'anglais n’a par exemple pas besoin de préciser dans les séquences déterminant + déterminé les rapports logiques qui unissent ces éléments. Aussi y rencontre-t-on habituellement des structures telles que :

(5) a. information referral system

b. a never thought-of solution

tandis que le français indique dans les structures de surface la nature de ces rapports au moyen de prépositions ou de subordonnées relatives :

(6) a. système d'orientation pour l'information

b. solution à laquelle on n'a jamais pensé ou solution (qui n'a) jamais (été) envisagée.

L'ordre des éléments au sein de ces syntagmes correspond à des structures sémantiques et syntactiques précises sur lesquelles nous ne reviendrons pas ici². Qu'il nous suffise de mentionner l'exemple suivant quelque peu caricatural mais acceptable en anglais :

(7) earth resources remote sensing satellite data collection platform qui correspond au syntagme français suivant :

(8) plate-forme de collecte de données recueillies par un satellite de télédétection des ressources terrestres

pour relever les contraintes sémantiques et syntactiques qui s'appliquent à ces syntagmes. Or, on constate qu'en français, les rapports qui unissent les composants de ces syntagmes au moyen d'éléments de jonction sont aussi implicites qu'en anglais, comme en témoignent les exemples suivants :

(9) a. appel longue distance (long distance call)

b. chaîne-or (gold chain, formé peut-être sur un exemple plus ancien :

c. étalon-or (gold standard)

d. image haute définition (high definition picture)

e. missile longue portée (long range missile)

L'hypothèse de l'anglicisation relève également que l'inversion de l'ordre des mots en français sur le modèle anglais est productif dans deux cas :

1. lorsque l'élément qui détermine le nom est considéré comme un composant savant c'est-à-dire assimilé à un emprunt au grec ou au latin. Il s'agit le plus souvent de troncations, de mots valises où l'ordre des éléments obéit à la règle, également en vigueur en anglais, selon laquelle le déterminant précède le déterminé. Aussi trouvonsnous :

(10) a. confisuc ou sucre spécial confitures

b. décoraries du Salon de la décoration (formé vraisemblablement sur floralies)

c. aromathérapie ou thérapie à base d'aromates

d. publigem pour publication de gemmes

e. créapolis pour cité de la création (où les deux éléments semblent relever de la composition savante)

2. dans la composition des noms propres et des marques commerciales :

(11) a. phénomène "Intelligence Energie Club"

b. Revillon Boutique

c. Rank Xerox Diffusion

Enfin, l'hypothèse de l'anglicisation fait valoir que nombre d'expressions nouvelles, d'ajouts ou de suppressions sont liés à des contraintes de l'anglais.

Parmi les expressions nouvelles incriminées se trouvent des syntagmes relevant de la langue commune tout comme des langues de spécialité. J. Darbelnet ${ }^{3}$ qualifie d'anglicismes de pensée les termes qui traduisent littéralement l'anglais :

(12) a. salad bar

b. Price Earning Ratio 
rendus plus souvent en français par les expressions suivantes :

(13) a. bar à salade

b. PER ou ration rapport/prix

que par des équivalents plus éloignés lexicalement et syntactiquement de l'anglais :
(14) a. buffet de crudités
b. coefficient de capitalisation des résultats ${ }^{4}$

Les ajouts de mots dans les syntagmes complexes du français sous l'influence de l'anglais sont particulièrement nombreux dans le domaine de la géographie physique et humaine :

(15) a. Mount Palomar Observatory

b. Apache Indians

c. River Kwai Bridge ou bridge over the River Kwai

sont traduits généralement en français par

(16) a. Observatoire du Mont Palomar

b. Indiens apaches

alors qu'il suffirait de dire en français :

(17) a. Observatoire du Palomar

b. les Apaches

c. Pont de la rivière Kwaï

Il est par exemple intéressant de noter qu'un locuteur français ne dirait pas :

mais bien

(18) a. Pont de la rivière Seine

b. Pont du fleuve Seine

(19) Pont de la Seine,

de même que les syntagmes nominaux suivants :

(20) a. the River Thames

b. New York City

deviennent en français plus par convention semble-t-il que par nécessité de compréhension :

(21) a. la Tamise ou le fleuve de la Tamise

b. la ville $d e$ New York

La suppression d'éléments de liaison dans les syntagmes nominaux complexes en français peut également être attribuée à l'influence de la syntaxe anglaise. Trois types de suppression peuvent essentiellement se présenter :

1. la suppression de conjonction de coordination comme en témoignent les exemples suivants :

(22) a. dialogues rencontres instantanés

b. architecte concepteur

c. train-autos-couchettes

2. la suppression de préposition ou d'éléments marquant les rapports de subordination des éléments qui déterminent, caractérisent ou qualifient le composant principal, ce dernier constituant la tête du mot composé ou du syntagme nominal complexe, comme l'indiquent les exemples ci-après :

(23) a. solution crédit

b. capacité mémoire

c. garantie "kilométrage illimité "

d. département Analyses d'investissement

e. contrat garantie reprise

f. témoin marche/arrêt

g. adaptateur secteur

h. transport rail-route

i. filière fibres optiques

j. crème douceur jour

k. baume beauté éclair

Citons aussi le cas où, a priori, il est difficile de déterminer s'il existe un rapport de coordination ou de subordination au sein du syntagme :

(24) baladeur " carte de crédit " 
3. le recours à l'ellipse qui permet d'omettre des mots que la connaissance de la langue et en particulier des règles syntaxiques permet de suppléer. Ce procédé, courant en anglais, semble être de plus en plus fréquent en français. Ainsi, si des expressions elliptiques telles que :

(25) a. functional protection

b. substance abuse

c. post-harvest equipment

sont acceptables dans la terminologie des Nations unies, elles ne peuvent être rendues telles quelles en français pour des raisons de compréhension, étant donné que des traductions littérales :

(26) a. protection fonctionnelle c. équipement postmessial b. abus de substances

sont beaucoup plus opaques que les expressions non elliptiques en français :

(27) a. protection des fonctionnaires dans l'exercice de leurs fonctions

b. abus de substances psychotropes

c. matériel d'entreposage et de transformation

il n'en reste pas moins vrai que les formules elliptiques sont de plus en plus courantes en français, à telle enseigne que nous trouvons :

(28) a. parc floral Vincennes (pour parc floral de Paris au Bois de Vincennes)

b. spécial Salon de l'auto (pour événement spécial organisé à l'occasion du Salon de l'auto ou bien offre spéciale à saisir au Salon de l'auto)

c. informations presse (pour informations à l'usage des membres de la presse, par exemple)

d. transferts aéroport/hôtel

Ces effacements d'éléments de jonction ne vont pas sans créer des ambiguïtés qui se traduisent souvent par des hésitations d'ordre grammatical qui, à leur tour, se traduisent par des hésitations d'ordre orthographique. Ainsi, si image satellite pour image reçue par satellite ne provoque plus en général d'hésitation en raison de la lexicalisation de ce syntagme, il n'en est pas de même de :

(29) a. données ordinateur(s)

b. zone basse(s) pression(s)

c. télévision couleur(s) ${ }^{6}$

Les hésitations d'ordre sémantique ne sont pas, elles non plus, négligeables si nous considérons le syntagme suivant trouvé récemment dans une annonce publicitaire ${ }^{7}$ :

(30) canapé Nautilus, trois places, en cuir Murphy vachette fleur rectifiée grainée

L'hypothèse selon laquelle le français évoluerait selon les règles qui lui seraient propres et non selon des règles empruntées à l'anglais s'appuie sur les faits de langue suivants :

1. Une fois entrées dans la langue française, les expressions anglaises ou pseudoanglaises y sont employées conformément aux règles du français. Ainsi, les premiers éléments d'un composé anglais employé au pluriel reçoivent généralement la marque du pluriel en français, ce qui est rarement le cas en anglais. On trouve ainsi en français les emprunts suivants :

(31) a. wines bars

b. euronotes facilities (ou euronotes facilités) ${ }^{8}$

alors qu'en anglais seul le déterminé prendrait la marque du pluriel :

(32) a. wine bars

b. euronote facilities 
2. L'emprunt direct à l'anglais peut n'être qu'un phénomène temporaire. Ainsi les syntagmes :

(33) a. niveau de vie

b. voyagiste ou

organisateur de voyages

sont venus remplacer les calques de l'anglais tant dans le domaine lexical (cf. l'exemple 34 a.) que dans le domaine syntactique et lexical (cf. 34 b.) :

(34) a. standard de vie (formé sur standard of living)

b. tour opérateur (formé sur tour operator)

3. L'ordre des mots au sein des syntagmes nominaux complexes français respecte les règles d'agencement des mots en français, et non pas celles de l'anglais. Peu de syntagmes semble-t-il contreviennent à la règle qui veut que le déterminé précède le déterminant en français comme le montrent les exemples suivants :

(35) a. zéro pâturage

(formé sur zero grazing)

b. auto-école

c. Sud-Liban

(formé sur driving school)

d. Sudestasie

(formé sur South Lebanon)

(formé sur South East Asia)

À cela deux raisons : d'une part la lexicalisation de ces syntagmes (un mot composé, on l'a vu dans les exemples 10 peut être considéré par les locuteurs comme relevant de la composition savante même si ses composants ne sont ni grecs ni latins) et d'autre part la productivité de ces types de syntagmes étant principalement limités au domaine des noms propres, ceux-ci ne sont pas considérés par les linguistes, à tort peutêtre, comme relevant du domaine de la grammaire mais sont plutôt repoussés dans le domaine extralinguistique de la mode et du jeu.

4. Il est facile de noter que les syntagmes nominaux français répondent à des exigences du français et ne sont pas toujours le produit d'un mimétisme anglais. Ainsi, dans le domaine de l'informatique, nous trouvons les exemples suivants :

(36) a. code à barres ou code-barres qui correspondent à l'anglais bar-code

b. lecteur optique à l'anglais bar-code wand

c. instruction complète à l'anglais direct execution bar code

d. clavigramme à l'anglais paper keyboard ${ }^{9}$

5. La réduction des syntagmes nominaux français par effacement des éléments de jonction correspond à une tendance du français que l'on rencontre dans des syntagmes où l'influence de l'anglais est plus difficile à démontrer ${ }^{10}$. Ainsi :

(37) l'acronyme PVD correspond au syntagme pays en voie de développement alors que le syntagme correspondant anglais developing country n'a pas donné lieu à un acronyme dans la langue anglaise.

(38) le phénomène de l'ellipse et de l'apposition en français est très fréquent : femme-femme, enfant-roi, coffre-fort ventouse, tringle porte-manteau.

En conclusion, nous pouvons constater qu'une mise en parallèle même très partielle des arguments pour ou contre une influence prépondérante de l'anglais en contact avec le français tant dans les langues de spécialité que dans la langue commune met en lumière une série de problèmes grammaticaux et lexicaux qui n'ont été que peu étudiés en linguistique d'une manière contrastive. Les études de sociolinguistique ou de sociologie du langage, aussi bien faites soient-elles, n'apportent pour l'heure que peu de réponses à ces questions cruciales en matière de fertilisation terminologique : y a-t-il augmentation ou diminution du nombre de règles produisant les syntagmes nominaux français? Pouvons-nous parler de fertilisation grammaticale du français sous l'influence de l'anglais ? Tout porte à croire qu'il y a eu un phénomème de syncrétisme qui s'est produit en français, ne permettant plus au linguiste de parler de règles d'apposition, de 
juxtaposition, d'abrègement et d'adjectivisation ${ }^{11}$ en ce qui concerne les éléments déterminant un nom que les grammaires traditionnelles dénomment "sortes d'étiquettes" pour " expliquer" les cas suivants :

(39) question mariage, côté affaires, boutons fantaisie, vélo modèle course, pyjama taille 40, lustre genre rouet, point de vue théâtre, etc.

Une étude plus approfondie des mécanismes de production de ces syntagmes peut seule nous aider à déterminer le rôle de l'anglais dans la grammaire du français.

Notes et bibliographie

1. Dans cet article anglais désigne aussi bien l'anglais britannique que l'anglais des États-Unis.

2. Cf. Jastrab, M. (en préparation) : Complex Nominals, thèse de Ph.D., New York University.

3. Cf. Darbelnet, J. (1983) : "La norme lexicale et l'anglicisme au Québec", dans la Norme linguistique, textes colligés et présentés par E. Bédard et J. Maurais, Paris, éd. Gouvernement du Québec et Le Robert, pp. 603-623.

4. Cf. projet d'arrêté relatif à l'enrichissement du vocabulaire économique et financier, ministère de l'Économie, des Finances et de la Privatisation, Paris. Cet arrêté préparé par une commission de terminologie ne fait qu'entériner un usage déjà établi.

5. Ce syntagme peut, sans référence extralinguistique, désigner aussi bien un baladeur qui sert en même temps de carte de crédit ou un baladeur de la taille d'une carte de crédit.

6. La Commission de l'harmonisation de l'orthographe au sein du Conseil international de la langue française s'attache à l'heure actuelle à préciser les règles orthographiques qui s'appliquent à ces syntagmes.

7. Publicité de Cuir Center parue à plusieurs reprises dans le Figaro Magazine de septembre à octobre 1986.

8. Cf. l'article de C. Dufloux paru dans le Figaro du 31 mars 1986, p. 13. Le Monde du 9-10 février 1986, p. 15, fait également état du mot facility «ligne de crédit" dans les expressions suivantes :

(1) Securitised Note Commitment Facility (SNCF)

(2) Revolving Underwriting Facilities ou RUF

(3) Multi-currency Option Facility ou MOF

illustrant bien les difficultés de traduction que suscite ce mot au sein de syntagmes nominaux complexes que le journaliste ne se hasarde même pas à transposer en français.

9. Cf. Agron, P. (1986) : " Fiches du Comité d'étude des termes techniques français " (suite), dans Questions de français vivant, $\mathrm{n}^{\mathbf{0}} 11,3^{e}$ trimestre, Bruxelles, Maison de la Francité, pp. 19-23.

10. Le français jusqu'au XVIe siècle construisait en asyndète (c'est-à-dire sans mots de liaison) les syntagmes où s'exprimait la notion de "complément de nom ", lorsque ce complément désignait une personne comme le fait remarquer $F$. Picoche (communication personnelle). J. Chaurand (communication personnelle à la suite de cette présentation) considère que la production des syntagmes nominaux où plusieurs éléments sont juxtaposés tient à une libération des potentialités de la langue.

11. Notamment dans les sous-langues de spécialité. 